\title{
Pandemia por COVID-19: proyección de impacto en la seguridad alimentaria de niños, niñas y adolescentes
}

\author{
COVID-19 pandemic: impact on food security of children and adolescents
}

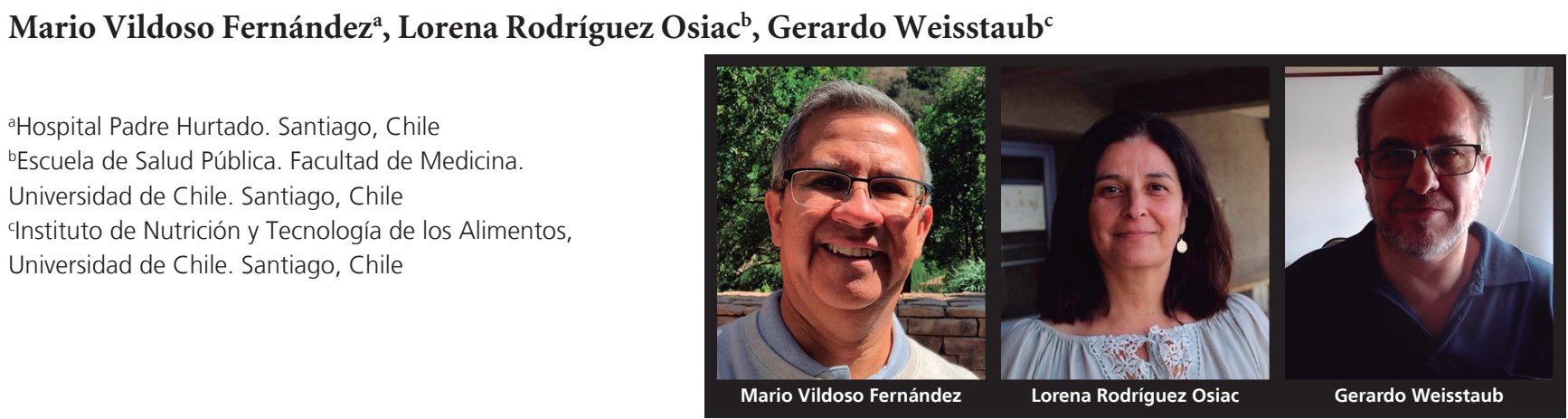

La nueva cepa de coronavirus ha infectado a más de 15 millones de personas en el mundo, 30 mil de las cuales son menores de edad chilenos ${ }^{1}$. Para proyectar el impacto posible de la pandemia en la alimentación de la población infantojuvenil en un país con el grado de inequidad como el nuestro ${ }^{2,3}$ se debe considerar el contexto en el que ésta ha ocurrido. Desde hace años que los niños, niñas y jóvenes de los sectores más pobres de la población, concentran la mayor prevalencia de problemas asociados a la alimentación y nutrición $n^{4}$. La pandemia además se instala en pleno desarrollo de la crisis social iniciada en octubre del 2019, lo que agrava su efecto sanitario, económico y alimentario, especialmente en la población más vulnerable ${ }^{5}$. Todavía es demasiado pronto para conocer en detalle el impacto del COVID-19 en la economía, ya que depende de muchas cosas que aún no sabemos (ej: cuánto durará la pandemia, que otras políticas públicas se pondrán en práctica para responder a la crisis). Lo que es muy probable, es que la recesión económica generada a nivel mundial tenga un mayor efecto en las economías en desarrollo. En estos contextos, una desaceleración en la economía puede agravar la inseguridad alimentaria existente.

En la Cumbre Mundial sobre la Alimentación (1996), los países acordaron que "Existe seguridad alimentaria cuando todas las personas tienen en todo momento acceso físico, social y económico a suficientes alimentos inocuos y nutritivos para satisfacer sus necesidades alimenticias y sus preferencias en cuanto a los alimentos a fin de llevar una vida activa y sana". En contraposición, el concepto de inseguridad alimentaria se refiere a la falta de uno o más de los elementos mencionados ${ }^{6}$. Para medir esta condición, el proyecto "Voices of the Hungry" desarrolló una Escala de Experiencia de Inseguridad Alimentaria (FIES por su sigla en inglés), que permite conocer la percepción a nivel individual y del hogar en una graduación que va desde la preocupación por tener o no tener alimentos, la calidad de los alimentos (saludables o no) y la escasez de alimentos en distinto grado, llegando hasta el sentir hambre (figura 1). Esta escala se aplica en más de 140 países del mundo, Chile entre ellos, y permite realizar comparaciones, planificar acciones y darles seguimiento y evaluación ${ }^{7}$.

Correspondencia:

Mario Vildoso Fernández

mariovildoso@gmail.com 
Figura 1. Grado de inseguridad alimentaria. Adaptado de "Métodos para la estimación de índices comparables de prevalencia de la inseguridad alimentaria experimentada por adultos en todo el mundo, FAO 2016".

\section{INSEGURIDAD ALIMENTARIA}

\begin{tabular}{|l|}
\hline PREOCUPACIÓN \\
ACERCA DE LA \\
CAPACIDAD DE \\
OBTENER \\
ALIMENTOS \\
\hline
\end{tabular}

CALIDAD Y

VARIEDAD DE

LOS ALIMENTOS

SE ENCUENTRA

COMPROMETIDA

\begin{tabular}{l}
\hline SE REDUCE LA \\
CANTIDAD DE \\
ALIMENTOS, SE \\
SALTAN \\
COMIDAS
\end{tabular}

SE PASA

HAMBRE
La encuesta CASEN incluye un módulo sobre seguridad alimentaria, en el que se pregunta si durante los últimos 12 meses en algún momento: a) ¿Usted u otra persona en su hogar se preocupó por no tener suficientes alimentos para comer por falta de dinero $\mathrm{u}$ otros recursos? b) ¿Alguna vez usted u otra persona en su hogar no pudo comer alimentos saludables y nutritivos por falta de dinero u otros recursos? c) Pensando en los últimos 12 meses, ¿alguna vez usted u otra persona en su hogar comió poca variedad de alimentos por falta de dinero u otros recursos? d) ¿Alguna vez usted u otra persona en su hogar tuvo que dejar de desayunar, almorzar, tomar once o cenar porque no había suficiente dinero u otros recursos para obtener alimentos? e) ¿Alguna vez usted u otra persona en su hogar comió menos de lo que pensaba que debía comer por falta de dinero u otros recursos? f) Pensando en los últimos 12 meses, ¿alguna vez su hogar se quedó sin alimentos por falta de dinero u otros recursos? g) ¿Alguna vez usted $\mathrm{u}$ otra persona en su hogar sintió hambre y no comió por falta de dinero $\mathrm{u}$ otros recursos para obtener alimentos? h) ¿Alguna vez usted u otra persona en su hogar dejó de comer todo un día por falta de dinero u otros recursos? ${ }^{8}$.

A 10 años de la meta "Hambre cero" de los "Objetivos de Desarrollo Sostenible" de las Naciones Unidas", el panorama de la inseguridad alimentaria en América Latina y el Caribe no es auspicioso, mostrando un notorio aumento de la inseguridad alimentaria moderada/grave y grave desde $26 \%$ y $7 \%$ en el trienio $2014-16$ a $31,7 \%$ y $9,4 \%$ en el período $2017-19^{10}$.

Aunque el problema en Chile es de menor magnitud, según la Encuesta CASEN 2017 el 13,1\% de los hogares en Chile presentan inseguridad alimentaria moderada/grave. Estas cifras empeoran significativamente si se consideran los hogares pobres $(32,2 \%)$, cuando la mujer es la jefa de hogar (16,3\%), cuando el jefe de hogar está desocupado (30,4\%), cuando alguno de sus integrantes presenta discapacidad $(17,7 \%)$ y en hogares con niñas, niños y adolescentes $(15,6 \%)$, en parti- cular si padecen de desnutrición (18,5\%). Cuando se consulta por la percepción de baja disponibilidad de alimentos responden afirmativamente el 16,8\% de los hogares pertenecientes a etnias y el $20,4 \%$ de aquellos formados por inmigrantes. La misma encuesta revela que 600.000 hogares $(3,4 \%)$ sufren inseguridad alimentaria grave ${ }^{8}$.

Considerando esta situación en nuestro país, es dable prever que la pandemia podría agravar la inseguridad alimentaria por lo que es indispensable tomar medidas nacionales y locales, especialmente dirigidas a los niños, niñas y adolescentes, y a la población más vulnerable. Preliminares datos de la Encuesta Social Covid-19 del Ministerio de Desarrollo Social realizada durante julio de 2020 muestran que casi $60 \%$ de los hogares declara haber disminuido sus ingresos, siendo más compleja la situación de los hogares liderados por mujeres y con presencia de niños, niñas y adolescentes; $54 \%$ dice haber disminuido sus gastos en alimentos y 19,4\% está enfrentando problemas de inseguridad alimentaria moderada/grave $\mathrm{e}^{11}$.

Existe información también de otros países que muestran que durante la pandemia aumenta la inseguridad alimentaria. En el área metropolitana de la Provincia de Buenos Aires (Argentina) el porcentaje de hogares con niñas/os y adolescentes en los que se redujo el acceso de alimentos durante la cuarentena por problemas económicos aumentó del 26\% al 30\%. El incremento fue aún más evidente al analizar los casos en que se detectó inseguridad alimentaria grave, situaciones de "hambre" por falta de alimentos, que aumentó del 6,5\% al 15\% en aquellos hogares con niños, niñas o adolescentes ${ }^{12}$.

El fortalecimiento de los programas de asistencia alimentaria y nutricional existentes en Chile desde hace más de 50 años, parece una medida obvia y fácil de realizar, puesto que la logística para ello está implementada y existen las instituciones a cargo que pueden ejecutarla. Pero estas medidas, deben ser complementadas con otras que les permitan a las familias 
evitar situaciones de inseguridad alimentaria tanto en la disponibilidad como en el acceso a una cantidad suficiente de alimentos de alta calidad nutricional, por ejemplo, con apoyo económico familiar, privilegiando el comercio en ferias libres, y potenciando cadenas cortas de comercialización y la organización comunitaria local en comedores y ollas comunes.

El riesgo que reaparezca la desnutrición en algunos grupos y que paradójicamente aumente la obesidad en otros, generando una doble carga de enferme$\mathrm{dad}^{13}$, es una realidad que se debe tener presente y que debe ser abordada con políticas y programas públicos y sociales.

Incorporar el derecho a la alimentación en la nueva constitución contribuirá a que el Estado cumpla con la "garantía de que los individuos, las familias y la comunidad en su conjunto, accedan en todo momento a suficientes alimentos inocuos y nutritivos, principalmente producidos en el país en condiciones de competitividad, sostenibilidad y equidad, para que su consumo y utilización biológica les procure una óptima nutrición, una vida sana y socialmente productiva, con respeto a la diversidad cultural y preferencias de los consumidores" 14 .

\section{Conflicto de intereses}

Los autores declaran no tener conflicto de intereses.

\section{Referencias}

1. Cifras Oficiales COVID-19. MINSAL (acceso agosto 2020 https://www.gob.cl/ coronavirus/cifrasoficiales/)

2. Sánchez H, Albala C. Inequalities in health. Adult mortality in communities of Metropolitan Santiago. Rev Med Chile 2004;132: 454-60.

3. Gallardo K, Varas L, Gallardo M. Inequality of opportunity in health: evidence from Chile. Rev Saude Publica. 2017; 4; 51:110. doi: 10.11606/S1518-

4. Núñez A, Manzano CA, Chi C. Health outcomes, utilization, and equity in Chile: an evolution from 1990 to 2015 and the effects of the last health reform. Public Health. 2020; 178:38-48. doi: 10.1016/j. puhe.2019.08.017.

5. Artaza Barrios O, Méndez CA. Crisis social y política en Chile: la demanda por acceso y cobertura universal de salud. Rev Panam Salud Publica. 2020; 44: e16. https://doi.org/10.26633/ RPSP.2020.16.
6. FAO. Cumbre Mundial de la Alimentación 1996 (acceso agosto 2020 http://www.fao.org/wfs/index_es.htm).

7. FAO. Métodos para la estimación de índices comparables de prevalencia de la inseguridad alimentaria experimentada por adultos en todo el mundo 2016 (http://www.fao.org/3/b-i4830s.pdf)

8. Ministerio de Desarrollo Social y Familia. Encuesta CASEN 2017. Inseguridad Alimentaria (acceso en agosto de $2020 \mathrm{http}: / /$ observatorio. ministeriodesarrollosocial.gob.cl/casenmultidimensional/casen/casen_2017.php)

9. Objetivos de Desarrollo Sostenible: Hambre Cero (acceso agosto 2020 (https://www.un.org/ sustainabledevelopment/es/hunger/).

10. FAO, IFAD, UNICEF, WFP and WHO. 2020. The State of Food Security and Nutrition in the World 2020. Transforming food systems for affordable healthy diets. Rome, FAO (accesso agosto 2020 https://doi.org/10.4060/ca9692en).

11. Encuesta Social Covid-19. Ministerio de Desarrollo Social (acceso septiembre 2020 http://observatorio. ministeriodesarrollosocial.gob.cl/layout/ doc/covid/RESUMEN_Encuesta_Social_ Covid-19_03.09.2020.pdf.

12. Tuñón I, Sánchez ME. Las infancias en tiempos de cuarentena. Observatorio de la Deuda Social Argentina Universidad Católica Argentina. Mayo 2020 (acceso en agosto de 2020 http://wadmin.uca.edu.ar/public/ ckeditor/Observatorio\%20Deuda\%20 Social/Presentaciones/2020/2020_ OBSERVATORIO_EDSA\%20COVID19_ INFANCIA-V.pdf).

13. Atalah E, Amigo H, Bustos P. Does Chile's nutritional situation constitute a double burden? Am J Clin Nutr 2014; 100 (suppl): 1623S-7S.

14. FAO. Ley Marco Derecho a la Alimentación, Seguridad y Soberanía Alimentaria. XVIII Asamblea Ordinaria del Parlamento Latinoamericano $30 \mathrm{de}$ noviembre al 1 de diciembre de 2012. (http://www.fao.org/3/a-au351s.pdf). 